\title{
Peripheral administration of selective GlyT2 inhibitor, oleoyl-D-lysine, is analgesic in neuropathic but not acute or inflammatory pain models in mice
}

\author{
Bruce Wilson ${ }^{1}$, Julian Peiser-Oliver ${ }^{1}$, Alexander Gillis ${ }^{1}$, Sally Evans ${ }^{1}$, Claudia Alamein ${ }^{1}$, \\ Shannon Mostyn ${ }^{1}$, Susan Shimmon ${ }^{2}$, Tristan Rawling ${ }^{2}$, Macdonald Christie ${ }^{1}$, Robert \\ Vandenberg $^{1}$, and Sarasa Mohammadi ${ }^{1}$ \\ ${ }^{1}$ The University of Sydney Faculty of Medicine and Health \\ ${ }^{2}$ University of Technology Sydney
}

September 25, 2021

\begin{abstract}
Background and Purpose: Changes to spinal glycinergic signalling are a feature of pain chronification. Normalising those changes by inhibiting glycine transporter-2 (GlyT2) is a promising treatment strategy. However, existing GlyT2 inhibitors e.g. ORG25543 are limited by narrow therapeutic windows and severe dose-limiting side effects such as convulsions, and are therefore poor candidates for clinical development. Experimental Approach: Analgesic and side-effect properties of intraperitoneally administered oleoyl-D-lysine, a lipid-based GlyT2 inhibitor, were characterised in mice. Analgesia was assessed in models of chronic neuropathic and inflammatory pain via the von Frey test, and acute nociception via hotplate. Side effects were scored via numerical rating scale, convulsions score, the Rotarod test and whole-body plethysmography for respiratory depression. Key Results: Oleoyl-D-lysine produced significant analgesia/anti-allodynia in the model for chronic neuropathic pain but not for chronic inflammatory or acute pain. No side effects were seen at the peak analgesic dose, $30 \mathrm{mg} \mathrm{kg}-1$. Mild side effects were observed at the highest dose, $100 \mathrm{mg} \mathrm{kg}-1$, in the numerical rating score, but no convulsions. These results contrasted markedly with ORG25543, which produced significant analgesia only at the lethal or near-lethal dose of $50 \mathrm{mg}$ kg-1. At this dose, ORG25543 caused severe side effects on the numerical rating score, severe convulsions, and Rotarod impairment. Oleoyl-D-lysine (30 mg kg-1) did not cause any respiratory depression, a problematic side effect of opiates. Conclusions and Implications: Oleoyl-D-lysine safely and effectively reverses neuropathic pain in mice. GlyT2 inhibitors may be better suited to treating pain of neuropathic origin over other pain aetiologies.
\end{abstract}

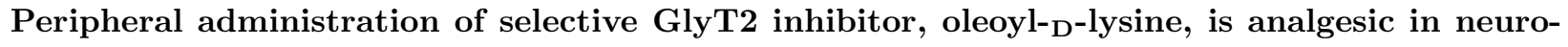
pathic but not acute or inflammatory pain models in mice

Inhibiting GlyT2 alleviates neuropathic but not inflammatory or acute pain

Bruce S Wilson ${ }^{\mathrm{a}}$, Julian Peiser-Oliver ${ }^{\mathrm{a}}$, Alexander Gillis ${ }^{\mathrm{a}}$, Sally Evans ${ }^{\mathrm{a}}$, Claudia Alamein ${ }^{\mathrm{a}}$, Shannon N Mostyn $^{\mathrm{a}}$, Susan Shimmon ${ }^{\mathrm{b}}$, Tristan Rawling ${ }^{\mathrm{b}}$, MacDonald J Christie ${ }^{\mathrm{a}}$, Robert J Vandenberg ${ }^{\mathrm{a}}$, Sarasa A Mohammadiat

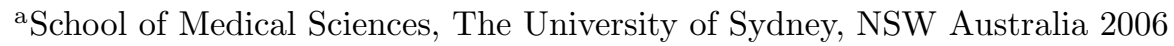

${ }^{\mathrm{b}}$ School of Mathematical and Physical Sciences, University of Technology, Sydney, NSW Australia 2006

Correspondence to: + Dr Sarasa Mohammadi, sarasa.mohammadi@sydney.edu.au Charles Perkins Centre, Level 3 West, the University of Sydney, NSW Australia 2006. 
Words: 2,950

Acknowledgements: Sincerest thanks to Dr Thomas Burton and the University of Sydney's Bosch Animal Behavioural Facility for practical training and assistance on the side effects tests.

Funding: NHMRC Project Grant APP144429. The funding source did not have any role in the study design, data collection, analysis, interpretation of data, or writing the report.

Conflict of interest statement: No conflicts of interest to report

\section{Bullet point summary:}

- What is already known:

- Partially inhibiting GlyT2 can alleviate chronic pain by restoring lost glycinergic function

- What this study adds:

- Novel lipid-based GlyT2 inhibitor, ol-D-lys, is safe and effective in alleviating neuropathic pain, but not inflammatory or acute pain

- Clinical significance :

- Clinical application of GlyT2 inhibitors may be better suited to neuropathic pain over other pain etiologies.

(Abstract)

Background and Purpose: Changes to spinal glycinergic signalling are a feature of pain chronification. Normalising those changes by inhibiting glycine transporter-2 (GlyT2) is a promising treatment strategy. However, existing GlyT2 inhibitors e.g. ORG25543 are limited by narrow therapeutic windows and severe dose-limiting side effects such as convulsions, and are therefore poor candidates for clinical development.

Experimental Approach: Analgesic and side-effect properties of intraperitoneally administered oleoyl- ${ }^{-}$ lysine, a lipid-based GlyT2 inhibitor, were characterised in mice. Analgesia was assessed in models of chronic neuropathic and inflammatory pain via the von Frey test, and acute nociception via hotplate. Side effects were scored via numerical rating scale, convulsions score, the Rotarod test and whole-body plethysmography for respiratory depression.

Key Results: Oleoyl-D-lysine produced significant analgesia/anti-allodynia in the model for chronic neuropathic pain but not for chronic inflammatory or acute pain. No side effects were seen at the peak analgesic dose, $30 \mathrm{mg} \mathrm{kg}^{-1}$. Mild side effects were observed at the highest dose, $100 \mathrm{mg} \mathrm{kg}^{-1}$, in the numerical rating score, but no convulsions. These results contrasted markedly with ORG25543, which produced significant analgesia only at the lethal or near-lethal dose of $50 \mathrm{mg} \mathrm{kg}^{-1}$. At this dose, ORG25543 caused severe side effects on the numerical rating score, severe convulsions, and Rotarod impairment. Oleoyl- ${ }^{-}$-lysine (30 mg $\mathrm{kg}^{-1}$ ) did not cause any respiratory depression, a problematic side effect of opiates.

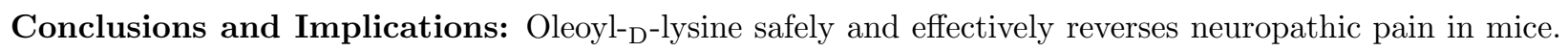
GlyT2 inhibitors may be better suited to treating pain of neuropathic origin over other pain aetiologies.

Keywords: Glycine transporter 2, analgesia, neuropathic pain, convulsions, mouse models

\section{INTRODUCTION}

Chronic pain causes terrible physical and psychological suffering that is often poorly managed by existing treatments. Neuropathic pain is the most difficult type of pain to treat. It results from damage or injury to the somatosensory nervous system and has a prevalence of approximately $18 \%$ of all chronic pain patients (von Hehn et al., 2012). Current pharmacotherapies such as non-steroidal anti-inflammatory drugs, opioids, anticonvulsants and antidepressants only provide clinically adequate analgesia in approximately $40 \%$ of sufferers (Breivik et al., 2006) and the utility of all are limited by adverse effects. Of particular concern, opioids are now a leading cause of unintentional death in the USA (Scholl et al., 2018; Kochanek et al., 2019), wherein overdose leads to fatal respiratory depression (Seth et al., 2018). 
In the search for improved pain relief options, the glycinergic system is being investigated for novel targets. Glycine is a major inhibitory neurotransmitter that maintains inhibitory tone in spinal pain pathways. In chronic neuropathic pain models, this tonic inhibition is compromised, causing disinhibition, enhanced pain signalling and inappropriate pain signals in response to non-noxious stimuli (allodynia; Figure 1; Imlach et al., 2016). Rodent models of chronic neuropathic pain show that a specific population of spinal dorsal horn lamina II interneurons (radial cells) lose inhibitory glycinergic input (Imlach et al., 2016), thus enhancing the efficacy of synaptic transmission of pain signals. Furthermore, intrathecal (i.t.) administration of glycine (Tanabe et al., 2008; Cheng et al., 2009), or inhibitors of glycine transporters (GlyT) (Hermanns et al., 2008; Morita et al., 2008; Mingorance-Le Meur et al., 2013; Motoyama et al., 2014; Takahashi et al., 2015) in rodents can restore the lost inhibitory signalling and attenuate pain responses (reviewed in (Dohi et al., 2009; Harvey and Yee, 2013; Vandenberg et al., 2014; Cioffi, 2018). Attenuation of pain responses was similarly observed after genetic knockdown of spinal GlyT expression in mice (Morita et al., 2008; Motoyama et al., 2014), thus establishing the spinal glycinergic system as a feasible target for analgesia. Glycine transporter 1 (GlyT1) is expressed mostly on glial cells throughout the brain and spinal cord and is involved in excitatory as well as inhibitory signalling. Glycine transporter 2 (GlyT2) is predominantly expressed on neurons in the spinal cord and brainstem; importantly, the highest densities of GlyT2 are on presynaptic glycinergic inhibitory neurons in lamina III of the dorsal horn (Zafra et al., 1995; Zeilhofer et al., 2005; Betz et al., 2006; Raiteri et al., 2008; Harvey and Yee, 2013; Aroeira et al., 2014; Schlösser et al., 2015; Cioffi, 2018). This distribution in a key location of the pain pathway indicates that GlyT2 is likely to be a promising target for analgesic drugs.

To date, the only blood-brain barrier permeable GlyT2 inhibitor with selectivity over GlyT1 that is commercially available is ORG25543 (Caulfield et al., 2001). However, on-target toxicity at analgesic doses limit its use in vivo (Mingorance-Le Meur et al., 2013) and safer alternatives are needed. Recently, a lipid-based GlyT2-selective allosteric inhibitor, oleoyl-D-Lysine (ol--D-lys), was developed based on the endogenous lipid, N-arachidonyl glycine (NAGly; Mostyn et al., 2019). Instead of a glycine amino acid head-group, a lysine head group in the ${ }_{\mathrm{D}}$-configuration was conjugated to an oleoyl tail to produce a metabolically stable, selective, reversible inhibitor of GlyT2. We formerly showed proof of principal analgesic efficacy of intraperitoneally (i.p.) administered ol-D-lys in rats (Mostyn et al., 2019). Here we undertook a detailed comparison of i.p. ol-D-lys and ORG-25543 in mice to comprehensively characterise the potential therapeutic value of ol- $\mathrm{D}^{-l y s}$ in multiple pain models.

\section{METHODS}

\section{Animals}

We used animal models for this project and have adhered to the ARRIVE and BJP guidelines in our reporting. We used well established and validated mouse models of pain that are ubiquitously used in pharmacological literature. This allows for meaningful comparisons across historical published studies and therefore serves as a reduction technique.

All studies were conducted in accordance with the Australian Code of Practice for the Care and Use of Animals for Scientific Purposes (8th edition, 2013). The study protocols were reviewed and approved by the University of Sydney Animal Ethics Committee (2018/1363).

Experiments were conducted on 357 male C57Bl/6 mice (ARC WA, Perth Australia), at 9 weeks of age and weighing $25-30 \mathrm{~g}$ on the day of testing. Mice were housed in a specific pathogen free facility maintained at $22 \pm 2^{\circ} \mathrm{C}$ and $40-70 \%$ humidity with lights on a $12 \mathrm{~h}$ light-dark cycle (06:00 - 18:00). Mice were housed in individually ventilated cages with corncob bedding, $\sim 4$ mice per cage (min 2, max 6$)$ and ad libitum access to food (irradiated mouse feed, Specialty Feeds, WA) and water. Standard enrichment of a nesting dome, nesting paper and wooden chew block was provided.

Animals were allowed 7 days acclimatisation to the research facility prior to commencement of any procedures and were then acclimatised to the experimenter (male experimenter for analgesia, side effects, and convulsions testing; female experimenter for plethysmography and rotarod), testing room, apparatus and handling for 
at least 4 days prior to experiments. Testing was carried out between the hours of 08:00 and 17:00.

A minimum of $n=6$ animals were used per group, except when a drug dose caused acute toxicity. A single cohort of mice was used to test the numerical rating scores and convulsion scores, while separate cohorts were tested for all other measures.

\section{Materials, drug preparation and administration}

Ol-D-lys (Mostyn et al., 2019) and ORG25543 (Tocris) were dissolved in a vehicle of 1\% DMSO (Sigma) and 10\% Solutol HS 15 (Sigma) in 0.9\% sterile saline. Morphine (Sigma) was dissolved in sterile saline only. A single bolus injection of $<10 \mathrm{ml} / \mathrm{Kg}$ volume was administered via i.p. route using 29-G needles (Terumo) and testing was performed at half-hourly to capture peak effect and then hourly or two-hourly to capture return to baseline.

Mice were randomly assigned to treatment groups using an online list randomiser (https://www.random.org/lists/) and pre-filled needles were labelled with the blinding code by an independent colleague. The experimenters were blind to treatment allocations for the duration of testing and blinding codes were revealed after data was gathered and ready for statistical analysis.

\section{Neuropathic pain model}

A chronic constriction injury (CCI) model was adapted from Benbouzid et al (2008); also in Mohammadi and Christie (2014). Under deep isoflurane anaesthesia (2-2.5\% saturated oxygen), the left sciatic nerve was isolated from surrounding tissue using blunt dissection. A $2 \mathrm{~mm}$ length of polyethylene tubing (Clay Adams $\Omega$ BD \& Co. Maryland; inner diameter $0.38 \mathrm{~mm}$, outer diameter $1.09 \mathrm{~mm}$ ), split lengthwise, was positioned around the sciatic nerve to produce uniform constriction in each animal. The skin incision was closed using tissue adhesive (3M Vetbond) and mice were returned to their home-cage to recover. Chronic neuropathic pain developed for 14 days before testing. Paw drooping or autotomy was not observed in any mice over the 14 days post-surgery.

\section{Inflammatory pain model}

The Complete Freund's Adjuvant (CFA) model of chronic inflammatory pain (Mohammadi and Christie, 2014; Pitzer et al., 2016) was performed via unilateral intra-plantar injection of $20 \mu \mathrm{L}$ of undiluted CFA using $25-\mathrm{G}$ needles. Injections were given under isoflurane anaesthesia (2-2.5\% saturated oxygen) and mice were given 3 days to recover from the procedure and to develop the chronic inflammatory pain state.

\section{Allodynia testing}

The von Frey test was used to assess the antiallodynic effects of GlyT2 inhibitors, as previously described (Mohammadi and Christie, 2014). Mice were placed on a mesh-floored chamber and allowed to acclimatise to the apparatus for 30 mins on the 2 days prior to baseline testing; for 20 minutes or until settled on the day of testing; and for approximately 10 minutes prior to each test time-point. A single von Frey filament (Stoelting Co. Chicago, IL) that elicited a $15 \%$ response rate in naïve mice, determined to be 0.4 gram-force (gf), was used. The filament was pressed to the plantar surface of the mouse's left hind-paw until it just bent. The response of the mouse was recorded as positive (vigorous hind-paw shake or licking of the hind-paw) or negative (no response). The percentage of responses to 10 separate applications of the von Frey hair were recorded as the animals' score. Von Frey thresholds were measured at baseline (pre-surgery), and 14-days post-surgery on the day of test (see section 1.1 for timing). Exclusion criteria of $>30 \%$ response rate at baseline or $<70 \%$ response rate at $\mathrm{t}=0$ were applied; no animals met these criteria.

\section{Acute pain testing (Hotplate)}

Acute pain was tested on a fixed temperature hotplate (IITC Life Sciences) set to $54^{\circ} \mathrm{C}$, with a $20 \mathrm{~s}$ maximum cut-off time. Mice were placed into Plexiglas chambers $(10 \mathrm{~cm} \mathrm{D} \mathrm{x} 15 \mathrm{~cm} \mathrm{H})$ with open top; a foot pedal initiated the timer the moment the hind-paws touched the hotplate and stopped the timer the moment 
an endpoint behaviour was observed; mice were then immediately removed from the hotplate. Endpoint behaviours were hind-paw shake or lick and jumping.

\section{Side effect testing}

Several tests for side effects were employed as listed below. Signs of other abnormal behaviours seen in GlyT2 knockout mice, such as impaired righting responses and hind-feet clasping (Gomeza et al., 2003) were absent and thus not quantified or reported here. These behaviours may be unique to early post-natal mice, with the homozygous knockout fatal around the second postnatal week, or only in cases of complete GlyT2 inhibition.

\section{Numerical rating score}

Side effects were scored using a severity rating scale from 0 to $3(0=$ no signs; $1=$ mild; $2=$ moderate; 3 = severe) that assessed 8 clinical behaviours; hypoxia, sedation, inactivity, sympathetic nervous hyperactivity (piloerection/shivering), pain/discomfort, dehydration, weak tone, convulsions. A mean score at each timepoint was generated for each drug dose.

\section{Convulsions score}

Motor convulsions have been reported following the administration of GlyT2 inhibitors in animal models of pain (Mingorance-Le Meur et al., 2013). A dedicated convulsions rating scale was used (Table 1). Average scores were generated for each mouse over the 6 -hour testing period.

\section{Motor incoordination; Rotarod test}

Possible motor incoordination caused by the drugs was tested using the Rotarod (IITC Life Science, CA, USA). Latency to fall from the rotarod was measured using a method adapted from Tung et al (2016). Briefly, mice were placed on rat-size rods (69.5 $\mathrm{mm}$ diameter, $27 \mathrm{~cm}$ drop to landing platform) and rotating speed accelerated from $4 \mathrm{rpm}$ to $40 \mathrm{rpm}$ with a maximum trial length of $180 \mathrm{sec}$. The Rotarod apparatus automatically detected the fall latency for each animal. Mice were given 5 minutes to acclimatise to the apparatus before each trial. A 3-day training period was given prior to baseline testing to allow acclimatisation to the test and eliminate any learning effects, wherein 3 training sessions were given at 30 min apart. During training, a trial was only counted if the mouse remained on the drum for a period of at least 10 seconds. If the staying period of 10 seconds was not met, the mouse was placed back on the rod and the trial was repeated.

\section{Respiratory depression}

Whole-body plethysmography (WBP) was used in freely moving mice to assess whether ol-D-lys causes respiratory side effects analogous to opioid analgesics. Naïve, uninjured mice were used for this test. Mice were placed into the WBP chamber (Buxco@, DSI, Harvard Bioscience, Inc) for 20 minutes to acclimatise, and baseline respiratory frequency was measured prior to drug administration. For each experimental timepoint, respiratory activity was measured for 10 minutes and averaged into 5-minute bins (Buxco Fineponte); the first bin was excluded from analysis as re-acclimation time, while the second bin was used in analysis. The change in respiratory frequency and minute volume (MV) as a percent of baseline was assessed as follows:

$$
\% \text { respiratory frequency }=\frac{\text { post injection }}{\text { pre injection }} * 100
$$

\section{Data and statistical analysis}

The data and statistical analysis comply with the recommendations on experimental design and analysis in pharmacology (Curtis et al., 2018). Data were analysed using GraphPad Prism (version 7.0b for Mac, GraphPad Software, La Jolla California USA, www.graphpad.com). Two-way repeated measures ANOVA was used where two independent factors (time and treatment) were compared. Where statistically significant main effects were observed, multiple comparisons with post-hoc Dunnett's correction was used, and statistically significant comparisons to vehicle control groups are reported. For data with non-normal distribution, 
the nonparametric Wilcoxon matched-pairs signed rank test was used. Non-normalised data are reported throughout, except for the rotarod and respiratory measures, where $\%$ of baseline is used to control for inter-individual variance at baseline. Data are represented as mean \pm SEM. Significance level was set at $p$ $=0.05$ and statistical significance is represented as $* \mathrm{p}<0.05$.

\section{RESULTS}

2. Analgesic effects of GlyT2-inhibitors

3. Chronic neuropathic pain

Systemic administration of ol-D-lys produced a significant analgesic effect in the CCI pain model (main effects of time $(\mathrm{F}(6,240)=35.26)$, treatment $(\mathrm{F}(5,40)=6.15)$ and an interaction effect $(\mathrm{F}(30,240)$ $=2.618)$ ). Post hoc analysis showed that the reversal of allodynia compared to vehicle was statistically significant at 60 minutes for 16,30 and $100 \mathrm{mg} \mathrm{kg}^{-1}$ doses, and with a peak effect reached at approximately 90 minutes. Significant effects of the $30 \mathrm{mg} / \mathrm{kg}$ dose and $100 \mathrm{mg} / \mathrm{kg}$ dose lasted for at least $6 \mathrm{~h}$. A dose response relationship was evident at lower doses, with a peak effect at $30 \mathrm{mg} \mathrm{kg}^{-1}$ (Figure 2A). Doses higher than $30 \mathrm{mg} \mathrm{kg}^{-1}$ lost the dose response relationship, with $50 \mathrm{mg} \mathrm{kg}^{-1}$ and $100 \mathrm{mg} \mathrm{kg}^{-1}$ producing only moderate or no analgesic effects compared with $30 \mathrm{mg} \mathrm{kg}^{-1}$. The analgesic efficacy of ol- $\mathrm{D}^{-1}$-ys contrasted with that of ORG25543, which also produced main effects of time $(\mathrm{F}(6,144)=7.09)$ and treatment $(\mathrm{F}(2,24)=3.877)$. ORG25543 similarly had a peak analgesic effect at 90 minutes (Figure 2B), but these analgesia scores are likely confounded by severe side effects (see below) and may not reflect a simple anti-allodynic effect. The maximum dose of ORG25543 tested was $50 \mathrm{mg} \mathrm{kg}^{-1}$. This dose was found to be lethal in one animal during side effect testing so for ethical reasons only $\mathrm{n}=3$ was tested here, and this dose was excluded from statistical analysis. For subsequent analgesia testing, only the sub-lethal dose of $30 \mathrm{mg} \mathrm{kg}^{-1}$ was used for ORG. This dose produced moderate analgesia from $1 \mathrm{~h}$, lasting for the full $6 \mathrm{~h}$ testing duration.

\section{Chronic inflammatory pain}

In the CFA pain model, 3, 30, and $100 \mathrm{mg} \mathrm{kg}^{-1}$ of ol-D-lys was tested over 6 hours, compared to vehicle or $30 \mathrm{mg} \mathrm{kg}{ }^{-1}$ of ORG25543. No significant analgesic effect was observed (significant main effect for time $(\mathrm{F}(6$, $204)=4.479$ ) but not for treatment or interaction) (Figure 3). To ensure that this apparent lack of effect was in fact a result of the pain model, rather than other variables, we separately conducted a small headto-head experiment comparing the effect of $30 \mathrm{mg} / \mathrm{kg}$ of ol-D-lys in CCI and CFA pain models compared to vehicle ( $\mathrm{n}=3$ per group), wherein the CCI model acted as positive control for the pain model. A peak effect

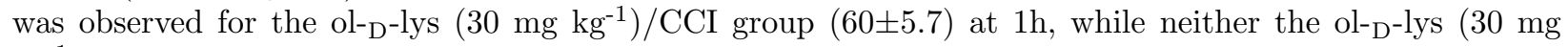
$\left.\mathrm{kg}^{-1}\right) / \mathrm{CFA}$ group $(90 \pm 5.7)$ nor the vehicle group (80 \pm 5.7$)$ showed significant analgesia at any time point.

\section{Acute pain}

Acute heat pain was not significantly alleviated by either GlyT2 inhibitors ol-D-lys or ORG25543 (Figure 4A), while the positive control, morphine, did produce near maximal anti-nociception, peaking at 30 mins. Non-parametric Kruskal-Wallis one-way test showed that morphine differed significantly from vehicle at 30 mins (Figure 4B). The ORG25532 group exhibited higher variability due to confounding side-effects in 2 animals, however, overall did not differ from the vehicle group.

\section{Side effects and convulsions from GlyT2-inhibitors}

Numerical rating scales of categorical endpoints were used to quantify the time-course and severity of the general side effects and the convulsions caused by GlyT2 inhibitors, ol--D-lys (Figure 5 A(i)-(ii), C) and ORG25543 (Figure $5 \mathrm{~B}(\mathrm{i})$-(ii), D(i)-(ii)) compared to the same behaviours pre-injection $(\mathrm{t}=0$ ). The $50 \mathrm{mg}$ $\mathrm{kg}^{-1}$ dose of ORG25543 was lethal in one animal (indicated by the cross symbol in Figures 5 B(i) and D(i)) so data is missing post 1-hour timepoint for one animal. Due to this toxicity, we did not attempt to test the full sample size for this dose on any measure.

Figure $5 \mathrm{~A}(\mathrm{i})$ and $\mathrm{B}(\mathrm{i})$ show the average time-course of onset and severity of side effects for $\mathrm{ol}^{-} \mathrm{D}-\mathrm{lys}$ and ORG25543 respectively, both having peak side effects at 1-hour post-injection. The nonparametric Wilcoxon matched-pairs signed rank test was used to compare the behaviours before and after injection for those doses 
that caused adverse side effects (Figures $5 \mathrm{~A}$ (ii) and B (ii)). The $100 \mathrm{mg} \mathrm{kg}^{-1}$ dose of ol-D-lys and the 50 $\mathrm{mg} \mathrm{kg}{ }^{-1}$ dose of ORG25543 both caused statistically significant side effects at $30 \mathrm{~min}, 60 \mathrm{~min}$ and $90 \mathrm{~min}$ post-injection.

Figure $5 \mathrm{C}$ and $\mathrm{D}(\mathrm{i})$ show the average time-course of onset and severity of convulsions for ol- $\mathrm{D}^{-l y s}$ and

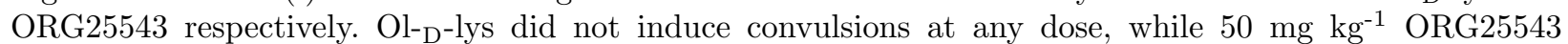
caused convulsions that peaked at 1-hour post-injection; the Wilcoxon signed rank test was again used to show that significant convulsions were observed at 30,60 and 90 minutes post-injection (Figure 5D(ii)).

\section{Motor coordination-effects of GlyT2-inhibitors}

The impact of GyT2 inhibitors on rotarod performance was assessed at various doses. For ol-D-lys, significant main effects of treatment $(\mathrm{F}(5,38)=2.783)$, time $(\mathrm{F}(5,190)=2.375)$, and an interaction between treatment and time $(\mathrm{F}(25,190)=1.843)$ were observed. While no detrimental effect of ol- ${ }_{\mathrm{D}}$-lys was observed at any dose, post-hoc tests showed an enhanced performance was observed for the $16 \mathrm{mg} \mathrm{kg}^{-1}$ dose at $1 \mathrm{~h}$ and $1.5 \mathrm{~h}$ (Figure 6A). For ORG25543, we again limited the number of animals tested at the higher dose of $50 \mathrm{mg} \mathrm{kg}^{-1}$ for ethical reasons and thus the analysis is underpowered. The two-way ANOVA showed only a main effect of time $(\mathrm{F}(5,125)=3.203)$ but not for treatment (Figure 6B). It would be necessary to collect a larger dataset to state with confidence whether $50 \mathrm{mg} \mathrm{kg}^{-1}$ ORG25543 had an effect on motor coordination.

\section{Respiratory effects of ol-D-Lys}

Respiratory depression is the primary cause of opioid-related deaths and has been reported to also be produced by ALX-1393, a nonselective GlyT2 inhibitor (Hermanns et al., 2008). In a comparison of ol-D-lys, morphine and vehicle, morphine produced significant respiratory depression as measured by both respiratory frequency (significant main effects of treatment $(\mathrm{F}(2,21)=6.883)$, time $(\mathrm{F}(5,105)=26.97)$ and a main interaction effect $(\mathrm{F}(10,105)=3.921)$ (Figure 7A) and MVb (significant main effects of treatment $(\mathrm{F}(2,21)$ $=12.93)$, time $(\mathrm{F}(5,105)=19.21)$ and a main interaction effect $(\mathrm{F}(10,105)=3.211)$ (Figure 7B). Multiple comparisons revealed that morphine caused statistically significant respiratory depression in both respiratory frequency and MV at multiple timepoints compared to vehicle. Ol---lys did not cause respiratory depression, and differed from vehicle only at 30 minutes, where respiratory frequency was slightly higher than vehicle.

\section{DISCUSSION and CONCLUSION}

GlyT2 inhibition holds promise as a new analgesic target, however severe side effects of compounds such as ORG25543 have tempered development. Here, we have shown that a reversible GlyT2 inhibitor is analgesic while eluding serious side effects. Detailed in vivo characterisation of $\mathrm{ol}_{-} \mathrm{D}^{-}$lys in mice showed it was an effective analgesic for neuropathic pain, with minimal side effects after systemic administration. We conducted a side-by-side comparison of ol-D-lys and ORG25543 - currently the only blood-brain barrier permeable, GlyT2-selective inhibitor commercially available. While useful as a research tool in vitro, the high toxicity of ORG25543 precludes its use in reliably characterising the in vivoeffects of inhibiting GlyT2. By virtue of its larger therapeutic window, we were able to test ol-D-lys in multiple pain and side-effect models and show that inhibition of GlyT2 appears to be a safe and beneficial approach to treating chronic neuropathic pain, but not chronic inflammatory pain or acute anti-nociception.

Ol-D-lys produced near complete reversal of allodynia in the mouse CCI neuropathic pain model. A dosedependent relationship was seen between 10 and $30 \mathrm{mg} \mathrm{kg}^{-1}$, while higher doses of 50 and $100 \mathrm{mg} \mathrm{kg}^{-1}$ were less effective. This may be due to the high lipophilicity and poor aqueous solubility of ol-D-lys leading to precipitate formation at higher doses, and/or the high degree of binding in the CNS, likely due to sequestration to lipid-rich brain tissue after crossing the blood-brain barrier (Mostyn et al., 2019). The analgesic effects of $30 \mathrm{mg} \mathrm{kg}^{-1}$ ORG25543 were modest but sustained for the $6 \mathrm{~h}$ duration of testing. At $50 \mathrm{mg} \mathrm{kg}^{-1}$, ORG25543 caused mice to reduce responding to the stimulus at every timepoint tested, however this is confounded by side effects, discussed below. Neither inflammatory pain nor acute thermal pain were alleviated by ol--D-lys or ORG25543. We propose that this is due to the underlying glycinergic changes that occur in chronic pain states, wherein loss of inhibitory glycinergic tone over time leads to increased nociceptive signalling. These 
changes are the target of GlyT2 inhibitors and may be absent in the shorter timescale CFA inflammatory model and acute pain model used here.

At the highest dose, ol-D-lys caused mild side effects on only one measure, while ORG25543 caused severe side effects as well as lethal toxicity. $\mathrm{Ol}_{-}{ }^{-}$-lys produced no convulsions at any dose. Mild to moderate clinical behaviours were scored for some mice on the numerical scale, but only at $100 \mathrm{mg} \mathrm{kg}^{-1}$; well above the peak analgesic dose. These behaviours included decreased activity, hunched posture, and mild pain behaviours of abdominal constriction, which suggest that a depot of the drug may have formed at the site of injection. Our prediction of precipitate formation at high doses supports this idea of a lipid depot forming and may account for the relatively low and sustained analgesic activity at $100 \mathrm{mg} \mathrm{kg}^{-1}$ (Zuidema et al., 1994). In contrast, ORG25543 produced severe side effects, convulsions and toxicity at $50 \mathrm{mg} \mathrm{kg}^{-1}$, with one mouse dying $1 \mathrm{~h}$ post-injection. Of those animals that were administered the $50 \mathrm{mg} \mathrm{kg}^{-1}$ dose, most reached the maximum (ceiling) score for the numerical rating scale, and one animal reached the ceiling for the convulsions score.

Ol-D-lys did not impede motor coordination in the rotarod test, with a significant effect only in the positive direction. The nature of such tests that involve subtle behavioural and motivational factors complicates interpretation of this result. Non-motor factors such as arousal and executive function (e.g. volition and motivation to stay on the rotarod; Yogev-Seligmann et al., 2008) may account for the behaviours, rather than drug-induced motor enhancementper se. With no other observable changes in this group at the time of testing, further experiments are required to determine the true cause of the enhanced rotarod performance. ORG25543 caused profound and immediate aberration of motor coordination in 2 out of 3 mice tested at $50 \mathrm{mg} \mathrm{kg}^{-1}$. However, due to the low numbers tested, this effect could not be statistically evaluated. The peak effects of ORG25543 on the numerical side-effect scale and convulsions score align temporally with the decrease in responses to the von Frey stimulus, thus, confounding the interpretation of the analgesic effects of ORG25543 at this dose. The von Frey test is highly sensitive to state of arousal, alertness and attention (Callahan et al., 2008). Furthermore, the von Frey test relies on discrete motor behaviours as endpoints for scoring pain thresholds, while ORG25543 caused motor incoordination. Thus, the reduced responding to von Frey stimuli likely reflects side effects such as convulsions preventing or distracting the mice from responding, not just analgesic effects of ORG25543.

Respiratory depression is a major side effect of opioids and has been shown to occur following administration of GlyT2 inhibitors (Hermanns et al., 2008). Here, we compared ol-D-lys with morphine, the current putative gold-standard analgesic drug for many types of pain. We observed no respiratory depression caused by the peak analgesic dose of ol-D-lys, $30 \mathrm{mg} \mathrm{kg}^{-1}$, compared with vehicle and an analgesic dose of morphine. A comparative increase in respiratory frequency, but not minute volume, at $30 \mathrm{~min}$ post-injection was observed. ORG25543 was not tested here due to ethical considerations, however these results support the proposal that reversible and selective GlyT2 inhibitors will not substantially affect respiration.

In this study, as we have previously shown in rats (Mostyn et al., 2019), we have observed severe side effects at minimally analgesic doses following i.p. delivery of ORG25543. Previous reports found complete reversal of allodynia at 100-fold lower doses of ORG25543 than in our study, and vastly differing pharmacodynamics (Morita et al., 2008; Motoyama et al., 2014). These past studies used a variety of pain models including a similar neuropathic pain model and the same von Frey test as used here. However, those studies found that the analgesic effects peaked from 3-24 h and lasted several days after a single bolus intravenous (i.v.) injection, with no side effects at analgesic doses. The lack of side effects may be due to the lower doses used compared to our study, although, Motoyama et al (2014) tested a single $1 \mathrm{mg} \mathrm{kg}^{-1}$ dose, orally (p.o.) administered, without reporting any side effects. There are two key points of difference between these reports and our findings; the route of administration (i.v. or p.o., compared with i.p.) and the vehicle (aCSF or saline, compared with $1 \%$ DMSO, $10 \%$ solutol in saline). While the route of administration may partially account for the differences in effective dose range, it is unlikely to account for this drastic pharmacodynamic difference. ORG25543 has good plasma and hepatic metabolic stability (Caulfield et al., 2001), thus hepatic metabolism upon i.p. administration is unlikely to account for the effects lasting for hours versus days. The vehicle used here was based on the requirements of dissolving the lipophilic ol-D-lys and may have affected the pharmacology of 
ORG25543. Indeed, an independent study (Mingorance-Le Meur et al. 2013) also used DMSO (5\%) in their vehicle and found that higher doses of ORG25543, $20 \mathrm{mg} \mathrm{kg}^{-1}$, were required to reach peak analgesic effect and observed convulsions and lethality at analgesic doses. Our findings indicate ORG25543 to have a narrow therapeutic window with severe on-target side effects at analgesic doses.

The utility of GlyT2 inhibitors as analgesics is being increasingly supported by pharmacological and genetic studies. There is evidence that irreversible blockers of GlyT2 (e.g. ORG25543; Mingorance-Le Meur et al., 2013) will lead to side effects such as convulsions and high toxicity, while reversible compounds (e.g. ol- ${ }^{-}$ lys) will have a larger therapeutic window. Additionally, the efficacy of compounds may influence side-effect liability, with full inhibitors causing more side effects than partial inhibitors. The difference is attributed to the role of GlyT2 in clearing synaptic glycine and repackaging it into presynaptic vesicles (Jursky and Nelson, 1995); complete depletion of vesicular glycine stores following complete, irreversible GlyT2 inhibition abolishes glycinergic signalling. However, partial or reversible inhibition of the transporter preserves vesicle repackaging while slowing down synaptic clearance and prolonging glycine receptor activation. This concept is mirrored in mouse genetic studies where global deletion of GlyT2 (compare to full/irreversible inhibitor) causes spasticity, tremor, inability to right, and death at post-natal week 2 (Gomeza et al., 2003), whereas partial knockdown of GlyT2 (compare to partial/reversible inhibitor) imparts analgesia with no apparent side effects (Morita et al., 2008; Motoyama et al., 2014).

The potential of targeting GlyT2 clinically is currently being established with the multi-target drug opiranserin (VVZ-149, Vivizon). Opiranserin is a novel analgesic drug, acting as antagonist at both GlyT2 and serotonin receptor 2A (5HT2A; Pang et al., 2012). Opiranserin has successfully completed recruitment for its first phase 3 clinical trial, with recruitment for a second trial underway (https://clinicaltrials.gov/ct2/results?term=vvz-149). All trials for opiranserin have been conducted/planned in post-operative pain. Our findings suggest that the best indication for a single-target GlyT2 inhibitor is in chronic pain states, where spinal glycinergic changes have become aberrant, and may be corrected with reversible GlyT2 inhibitors. The present results also build evidence that reversible GlyT2 inhibitors such as ol-D-lys elude side effects observed with irreversible GlyT2 inhibition.

\section{References}

Aroeira, R.I., Sebastião, A.M., and Valente, C.A. (2014). GlyT1 and GlyT2 in brain astrocytes: Expression, distribution and function. Brain Struct. Funct. $219: 817-830$.

Benbouzid, M., Pallage, V., Rajalu, M., Waltisperger, E., Doridot, S., Poisbeau, P., et al. (2008). Sciatic nerve cuffing in mice: A model of sustained neuropathic pain. Eur. J. Pain 12 : 591-599.

Betz, H., Gomeza, J., Armsen, W., Scholze, P., and Eulenburg, V. (2006). Glycine transporters: Essential regulators of synaptic transmission. Biochem. Soc. Trans. 34 : 55-58.

Borges, K., Gearing, M., McDermott, D.L., Smith, A.B., Almonte, A.G., Wainer, B.H., et al. (2003). Neuronal and glial pathological changes during epileptogenesis in the mouse pilocarpine model. Exp. Neurol.182 : 2134 .

Breivik, H., Collett, B., Ventafridda, V., Cohen, R., and Gallacher, D. (2006). Survey of chronic pain in Europe: Prevalence, impact on daily life, and treatment. Eur. J. Pain $10: 287-333$.

Callahan, B.L., Gil, A.S.C., Levesque, A., and Mogil, J.S. (2008). Modulation of Mechanical and Thermal Nociceptive Sensitivity in the Laboratory Mouse by Behavioral State. J. Pain $9: 174-184$.

Caulfield, W.L., Collie, I.T., Dickins, R.S., Epemolu, O., McGuire, R., Hill, D.R., et al. (2001). The first potent and selective inhibitors of the glycine transporter type 2 [1]. J. Med. Chem. 44 : 2679-2682.

Cheng, W., Yin, Q., Cheng, M.Y., Chen, H.S., Wang, S., Feng, T., et al. (2009). Intracerebroventricular or intrathecal injection of glycine produces analgesia in thermal nociception and chemical nociception via glycine receptors. Eur. J. Pharmacol. 614 : 44-49. 
Cioffi, C.L. (2018). Modulation of glycine-mediated spinal neurotransmission for the treatment of chronic pain. J. Med. Chem.61 : 2652-2679.

Curtis, M.J., Alexander, S., Cirino, G., Docherty, J.R., George, C.H., Giembycz, M.A., et al. (2018). Experimental design and analysis and their reporting II: updated and simplified guidance for authors and peer reviewers. Br. J. Pharmacol. 175 : 987-993.

Dohi, T., Morita, K., Kitayama, T., Motoyama, N., and Morioka, N. (2009). Glycine transporter inhibitors as a novel drug discovery strategy for neuropathic pain. Pharmacol. Ther. 123 : 54-79.

Gomeza, J., Ohno, K., Hülsmann, S., Armsen, W., Eulenburg, V., Richter, D.W., et al. (2003). Deletion of the mouse glycine transporter 2 results in a hyperekplexia phenotype and postnatal lethality. Neuron 40 :.

Harvey, R.J., and Yee, B.K. (2013). Glycine transporters as novel therapeutic targets in schizophrenia, alcohol dependence and pain. Nat. Rev. Drug Discov. 12 : 866-885.

Hehn, C.A. von, Baron, R., and Woolf, C.J. (2012). Deconstructing the Neuropathic Pain Phenotype to Reveal Neural Mechanisms. Neuron 73 : 638-652.

Hermanns, H., Muth-Selbach, U., Williams, R., Krug, S., Lipfert, P., Werdehausen, R., et al. (2008). Differential effects of spinally applied glycine transporter inhibitors on nociception in a rat model of neuropathic pain. Neurosci. Lett. $445: 214-219$.

Imlach, W.L., Bhola, R.F., Mohammadi, S.A., and Christie, M.J. (2016). Glycinergic dysfunction in a subpopulation of dorsal horn interneurons in a rat model of neuropathic pain. Sci. Rep. 6 : 1-14.

Jursky, F., and Nelson, N. (1995). Localization of Glycine Neurotransmitter Transporter (GLYT2) Reveals Correlation with the Distribution of Glycine Receptor. J. Neurochem. 64 : 1026-1033.

Kochanek, K.D., Murphy, S.L., Xu, J., and Arias, E. (2019). Deaths: Final data for 2017. Natl. Vital Stat. Reports $68: 1-18$.

Mingorance-Le Meur, A., Ghisdal, P., Mullier, B., Ron, P. De, Downey, P., Perren, C. Van Der, et al. (2013). Reversible inhibition of the glycine transporter GlyT2 circumvents acute toxicity while preserving efficacy in the treatment of pain. Br. J. Pharmacol. 170 : 1053-1063.

Mohammadi, S., and Christie, M.J. (2014). A9-Nicotinic Acetylcholine Receptors Contribute To the Maintenance of Chronic Mechanical Hyperalgesia, But Not Thermal or Mechanical Allodynia. Mol. Pain10 : $1-9$.

Morita, K., Motoyama, N., Kitayama, T., Morioka, N., Kifune, K., and Dohi, T. (2008). Spinal antiallodynia action of glycine transporter inhibitors in neuropathic pain models in mice. J. Pharmacol. Exp. Ther.326 : 633-645.

Mostyn, S.N., Rawling, T., Mohammadi, S., Shimmon, S., Frangos, Z.J., Sarker, S., et al. (2019). Development of an N-Acyl Amino Acid That Selectively Inhibits the Glycine Transporter 2 to Produce Analgesia in a Rat Model of Chronic Pain. J. Med. Chem. 62 : 2466-2484.

Motoyama, N., Morita, K., Shiraishi, S., Kitayama, T., Kanematsu, T., Uezono, Y., et al. (2014). Relief of cancer pain by glycine transporter inhibitors. Anesth. Analg. 119: 988-995.

Pang, M.H., Kim, Y., Jung, K.W., Cho, S., and Lee, D.H. (2012). Foundation review: A series of case studies: Practical methodology for identifying antinociceptive multi-target drugs. Drug Discov. Today 17 : 425-434.

Pitzer, C., Kuner, R., and Tappe-Theodor, A. (2016). Voluntary and evoked behavioral correlates in neuropathic pain states under different social housing conditions. Mol. Pain 12 : 1-12. 
Raiteri, L., Stigliani, S., Usai, C., Diaspro, A., Paluzzi, S., Milanese, M., et al. (2008). Functional expression of release-regulating glycine transporters GLYT1 on GABAergic neurons and GLYT2 on astrocytes in mouse spinal cord. Neurochem. Int. 52 : 103-112.

Schlösser, L., Barthel, F., Brandenburger, T., Neumann, E., Bauer, I., Eulenburg, V., et al. (2015). Glycine transporter GlyT1, but not GlyT2, is expressed in rat dorsal root ganglion-Possible implications for neuropathic pain. Neurosci. Lett. $600: 213-219$.

Scholl, L., Seth, P., Kariisa, M., Wilson, N., and Baldwin, G. (2018). Drug and Opioid-Involved Overdose Deaths — United States, 2013-2017. MMWR. Morb. Mortal. Wkly. Rep. 67 : 2013-2017.

Seth, P., Rudd, R.A., Noonan, R.K., and Haegerich, T.M. (2018). Quantifying the epidemic of prescription opioid overdose deaths. Am. J. Public Health 108 : 500-502.

Takahashi, Y., Hara, K., Haranishi, Y., Terada, T., Obara, G., and Sata, T. (2015). Antinociceptive effect of intracerebroventricular administration of glycine transporter-2 inhibitor ALX1393 in rat models of inflammatory and neuropathic pain. Pharmacol. Biochem. Behav.130 : 46-52.

Tanabe, M., Takasu, K., Yamaguchi, S., Kodama, D., and Ono, H. (2008). Glycine transporter inhibitors as a potential therapeutic strategy for chronic pain with memory impairment. Anesthesiology 108 : 929-937.

Tung, V.W.K., Burton, T.J., Quail, S.L., Mathews, M.A., and Camp, A.J. (2016). Motor performance is impaired following vestibular stimulation in ageing mice. Front. Aging Neurosci. $8: 1-10$.

Vandenberg, R.J., Ryan, R.M., Carland, J.E., Imlach, W.L., and Christie, M.J. (2014). Glycine transport inhibitors for the treatment of pain. Trends Pharmacol. Sci. 35 : 423-430.

Yogev-Seligmann, G., Hausdorff, J.M., and Giladi, N. (2008). The role of executive function and attention in gait. Mov. Disord. 23 :.

Zafra, F., Aragón, C., Olivares, L., Danbolt, N.C., Giménez, C., and Storm-Mathisen, J. (1995). Glycine Cells Are Differentially Expressed CNS in the spinal. J. Neurosci. 15 : 3952-3969.

Zeilhofer, H.U., Studler, B., Arabadzisz, D., Schweizer, C., Ahmadi, S., Layh, B., et al. (2005). Glycinergic neurons expressing enhanced green fluorescent protein in bacterial artificial chromosome transgenic mice. J. Comp. Neurol. 482 : 123-141.

Zuidema, J., Kadir, F., Titulaer, H.A.C., and Oussoren, C. (1994). Release and absorption rates of intramuscularly and subcutaneously injected pharmaceuticals (II). Int. J. Pharm. 105 : 189-207.

Table 1. Motor convulsion scale (from Borges et al., 2003)

\begin{tabular}{ll}
\hline Stage & Behaviour \\
\hline 0 & Normal activity \\
1 & Rigid posture or immobility \\
2 & Stiffened, extended and often arched tail \\
3 & Partial body clonus, including forelimb or hindlimb clonus (seen rarely) or head bobbing \\
3.5 & Whole body continuous clonic seizures while retaining posture \\
4 & Rearing \\
4.5 & Severe whole body continuous clonic seizures while retaining posture \\
5 & Rearing, falling \\
6 & Tonic-clonic seizures, loss of posture, jumping \\
\hline
\end{tabular}

Figure 1. Glycine inhibition in the dorsal horn is lost in chronic pain states, but may be restored by GlyT2 inhibitors to alleviate pain: Glycinergic inhibition to lamina II excitatory interneurons is lost in chronic pain states, leading to enhancement of nociceptive signals, and aberrant activation of the 
nociceptive pathways in response to non-noxious stimuli such as touch (via A $\beta$ fibres). Drugs that rectify this glycinergic dysfunction, such as GlyT2-inhibitors, are able to alleviate pain by inhibiting the abnormal signalling.

Figure 2. Analgesia (von Frey) testing in a mouse model of chronic neuropathic (CCI) pain: A Ol-D-lys (purple) produced analgesia in mice in a dose-dependent manner from $10 \mathrm{mg} \mathrm{kg}{ }^{-1}$ to $30 \mathrm{mg} \mathrm{kg}^{-1}$ at which a peak effect was seen. Higher doses of $50 \mathrm{mg} \mathrm{kg}^{-1}$ and $100 \mathrm{mg} \mathrm{kg}^{-1}$ were less effective than the lower dose of $30 \mathrm{mg} \mathrm{kg}^{-1}$. B ORG25543 produced statistically significant analgesia from $60 \mathrm{~min}$ to $4 \mathrm{~h}$ at the $30 \mathrm{mg} \mathrm{kg}^{-1}$ dose. At the highest tested dose of $50 \mathrm{mg} \mathrm{kg}^{-1}$, responses to the von Frey stimuli were markedly reduced, however this coincided with severe side effects and toxicity, which precluded testing the full data set and this dose was excluded from statistical analysis. Significant post-hoc multiple comparisons are indicated by asterisks $(* p<0.05)$. n's per group are indicated in parentheses in legend.

Figure 3. Analgesia (von Frey) testing in a mouse model of inflammatory (CFA) pain: No analgesia was observed for any dose of ol-D-lys or for $30 \mathrm{mg} \mathrm{kg}^{-1}$ of ORG25543. n's per group are indicated in parentheses in legend.

Figure 4. Acute pain (hotplate) testing in mice: No anti-nociception was observed for $30 \mathrm{mg} \mathrm{kg}^{-1}$ of ol-D-lys or for $30 \mathrm{mg} \mathrm{kg}^{-1}$ of ORG25543 while non-parametric Kruskal-Wallis one-way test showed significant anti-nociception at 30-min in the morphine (orange, $10 \mathrm{mg} \mathrm{kg}^{-1}$ ) group $(* \mathrm{p}<0.05$ ). n's per group are indicated in parentheses in legend.

Figure 5. Side effects and convulsions caused by GlyT2-inhibitors: A(i)-(ii) show side effects of ol-D-lys. B(i)-(ii) show side effects of ORG25543. C shows convulsions caused by ol-D-lys. D (i)-(ii) convulsions caused by ORG25543. Summary shows mean +/- SEM. Panel (ii) of A, B and D show the scatter of the doses that caused significant adverse behaviours compared to $t=0$, for which Wilcoxon signed rank test was performed $(* \mathrm{p}<0.05)$. The cross marked after 1 hour in $\mathrm{B}(\mathrm{i})$ and $\mathrm{D}(\mathrm{i})$ indicates the time at which one animal died. n's per group are indicated in parentheses in legend.

Figure 6. Motor coordination Rotarod test: AOl-${ }_{-}$-lys caused no dose-dependent motor incoordination. However, an enhanced performance was observed at 60 and 90 minutes, at the $16 \mathrm{mg} \mathrm{kg}^{-1} \mathrm{group}$ performed $(* \mathrm{p}<0.05)$.B ORG25543 caused profound and immediate incoordination in 2 out of 3 animals at $50 \mathrm{mg} \mathrm{kg}{ }^{-1}$, however due to the low numbers tested, this could not be statistically analysed. n's per group are indicated in parentheses in legend.

Figure 7. Respiratory side effects: Whole body plethysmography of freely moving mice showed that a sub-maximal analgesic dose of morphine (orange) caused respiratory depression, measured by respiratory frequency and minute volume $(\mathrm{MVb})$, but ${ }^{-}{ }_{-}-$-lys did not. Significant post-hoc multiple comparisons are indicated by asterisks $\left({ }^{*} p<0.05\right)$. n's per group are indicated in parentheses in legend. 






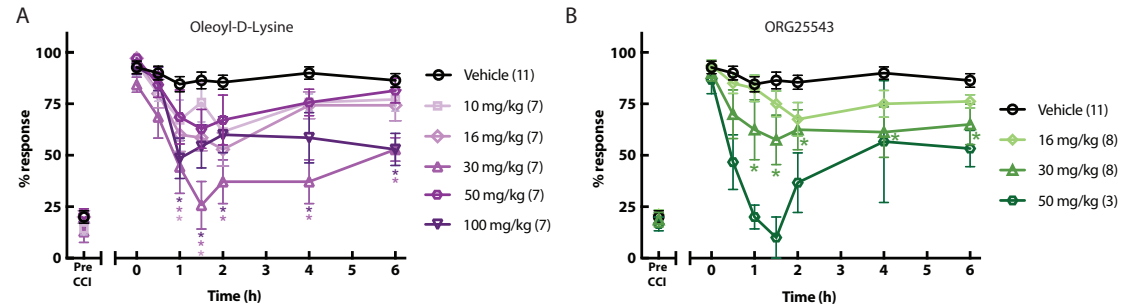


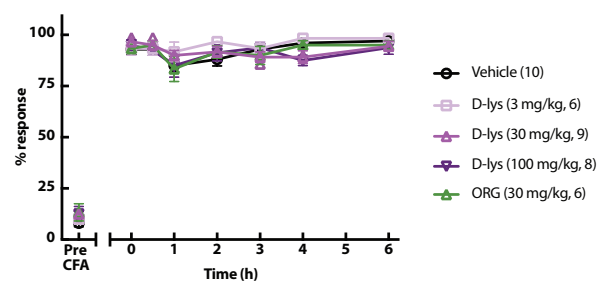



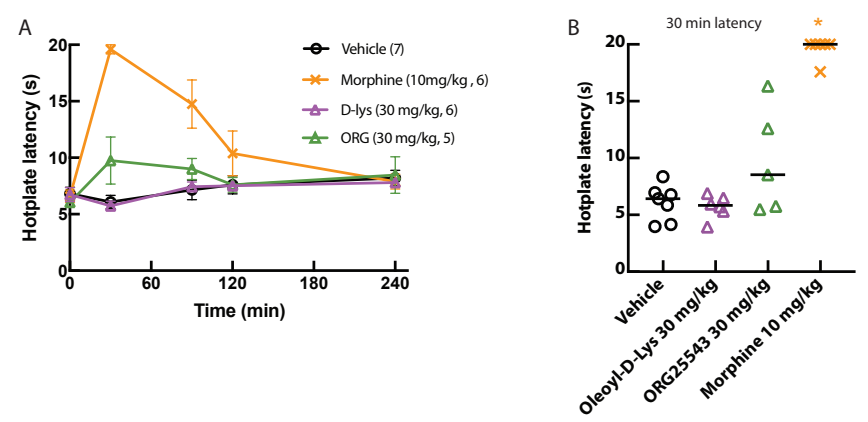

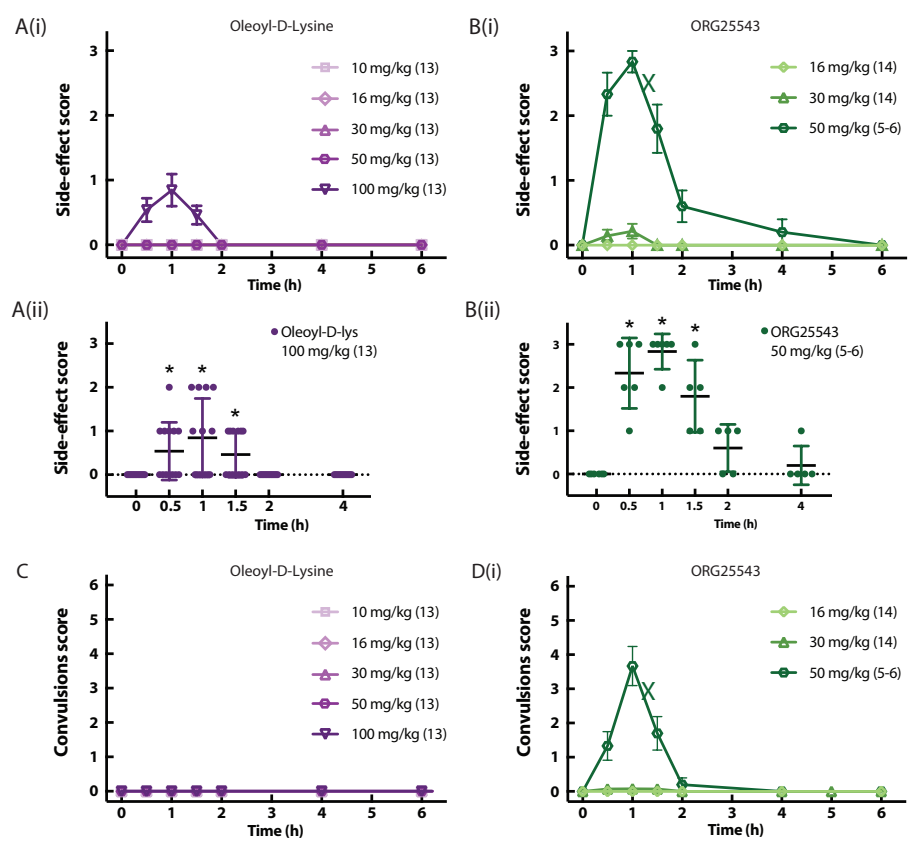

B(ii)
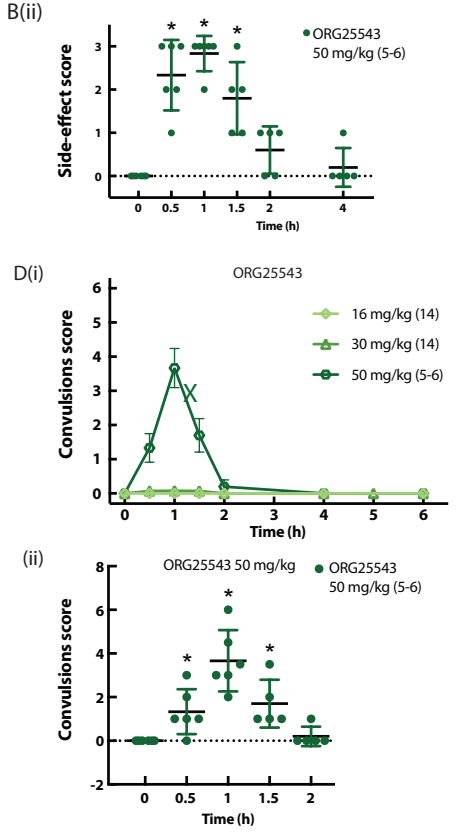

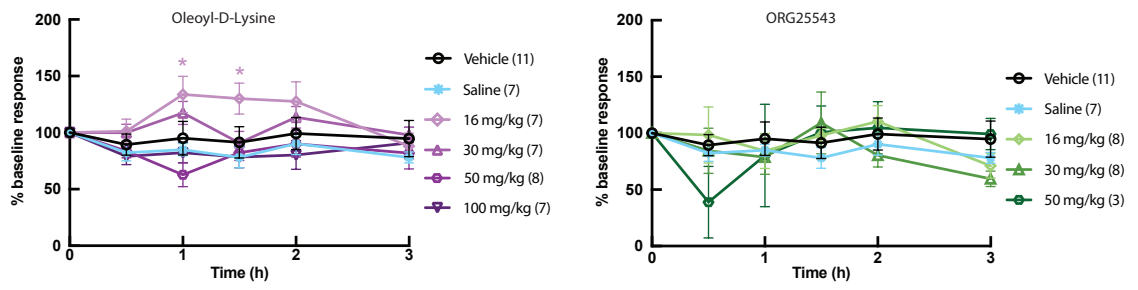

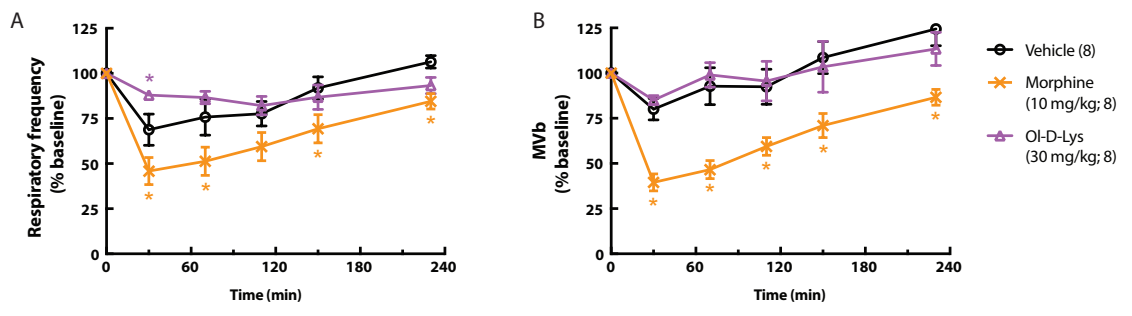